\title{
Cobertura populacional do Sistema de Vigilância Alimentar e Nutricional no Estado de São Paulo, Brasil
}

\author{
Population coverage of the food and nutrition surveillance system \\ in the state of São Paulo, Brazil
}

Carla Cristina Enes ${ }^{1}$

Helena Loiola ${ }^{2}$

Maria Rita Marques de Oliveira ${ }^{3}$

${ }^{1}$ Faculdade de Nutrição, Pontifícia Universidade Católica de Campinas. Av. John Boyd Dunlop S/N Campus II, Jardim Ipaussurama. 13.060-904 Campinas SP Brasil. cacenes@gmail.com

${ }^{2}$ Universidade Federal de São Carlos.

${ }^{3}$ Instituto de Biociências, Universidade Estadual Paulista Julio de Mesquita Filho.

\begin{abstract}
This article sets out to estimate the population coverage of the Food and Nutrition Surveillance System (SISVAN) at different phases of life and to evaluate its operation in the state of Sao Paulo. The study included 65 cities divided in 14 state regions. The coverage of SISVAN was estimated using data from nutritional status monitoring available in public reports and on government websites, as well as the number of users attended by the public health services. Total users of public health services were obtained by the difference between the total number of inhabitants of 65 cities and the number of beneficiaries of private health plans. Most regions presented reduced population coverage $(<10 \%)$. Approximately $57 \%$ revealed population coverage of between 5 and $10 \%$. There was a preponderance of records of the nutritional status of children for all regions of the state. A startling statistic was the low coverage for the elderly population, which was zero or near zero in most regions. Despite the efforts by the government to expand and enhance SISVAN, nutritional monitoring in Sao Paulo is still insufficient. This condition hinders the use of SISVAN to produce effective changes in food and nutrition policies.
\end{abstract}

Key words Food and nutrition surveillance system, Population coverage, Monitoring, Information systems, Nutritional status, Data quality
Resumo O objetivo deste artigo é estimar a cobertura populacional do Sistema de Vigilância Alimentar e Nutricional (SISVAN) nos diferentes estágios de vida e avaliar seu funcionamento no estado de São Paulo. O estudo incluiu 65 municípios divididos em 14 regiões do estado. A cobertura do SISVAN foi estimada a partir de dados de monitoramento do estado nutricional disponíveis nos relatórios públicos, e do número de usuários que frequentam os serviços públicos de saúde. O total de usuários foi obtido pela diferença entre o total de habitantes e o número de beneficiários de planos de saúde privados. A maioria das regiões apresentou uma cobertura reduzida $(<10 \%)$. Cerca de $57 \%$ revelaram cobertura entre 5 e 10\%. Constatou-se uma preponderância de registros do estado nutricional de crianças para todas as regiões do Estado. Chama a atenção a reduzida cobertura entre os idosos, que é inexistente ou próxima de zero na maioria das regiões. Apesar dos esforços empreendidos pelo governo visando à ampliação e à qualificação do SISVAN, o monitoramento nutricional no estado de São Paulo ainda é insuficiente. Esta condição compromete sua utilização na elaboração de políticas efetivas na área de alimentação e nutrição.

Palavras-chave Sistema de vigilância alimentar e nutricional, Cobertura populacional, Monitoramento, Sistemas de informação, Estado nutricional, Qualidade de dados 


\section{Introdução}

Entre as principais propostas de mudança na organização das práticas de saúde, na busca por um modelo de atenção integral aos indivíduos, destaca-se a vigilância, que propõe uma transformação do saber e das práticas por meio da redefinição do objeto, da reorientação do processo de trabalho e da reorganização dos serviços de saúde ${ }^{1}$.

Dentre os instrumentos que compõem a vigilância da saúde tem-se a vigilância alimentar e nutricional que é composta por um conjunto de estratégias da vigilância epidemiológica, que fornece dados sobre o perfil alimentar e nutricional da população com o intuito de subsidiar decisões políticas voltadas para a melhoria da situação de saúde².

Diferentemente da maioria dos indicadores clássicos de morbidade e mortalidade, as informações sobre o estado nutricional da população representam um indicador positivo de saúde. Entende-se por Vigilância Alimentar e Nutricional como sendo a descrição contínua e a predição de tendências das condições de alimentação e nutrição da população, bem como de seus fatores determinantes. Esta ação abrange ativi-dades de rotina, coletas e análises de dados e informações para descrever as condições alimentares e nutricionais da população ${ }^{3}$.

No Brasil, embora tenham sido descritas experiências incipientes e localizadas de vigilância nutricional desde meados da década de 1970, o Sistema de Vigilância Alimentar e Nutricional (SISVAN) foi implantado de maneira mais contundente, em nível nacional, somente a partir da década de 1990. Nesta ocasião o SISVAN foi instituído pelo Ministério da Saúde por meio da Portaria 1.156 de 31/08/1990 e, a partir da Lei Orgânica do Sistema Único de Saúde (Lei n ${ }^{\circ} 8.080$, de 19/09/1990, artigos $3^{\circ}, 6^{\circ}$ e $12^{\circ}$ ), foi recomendada a adoção do sistema no âmbito do Sistema Único de Saúde (SUS) ${ }^{4}$.

Desde 1993, com a criação do "Leite é Saúde", o SISVAN vem sendo vinculado a programas de alimentação e nutrição, sendo pré-requisito para a habilitação do município, a implantação do Sistema de Vigilância Alimentar e Nutricional. O programa de Incentivo ao Combate às Carências Nutricionais (ICCN) criado em 1998, também previa a vigilância nutricional como pré-requisito para a adesão ao mesmo e para o repasse de recursos federais ${ }^{5}$. O principal critério de permanência do município no programa era o envio regular de informações aos estados que pos- teriormente eram encaminhadas às instâncias específicas de vigilância alimentar e nutricional do Ministério da Saúde.

No início do ano 2000, um trabalho conjunto de representantes das Coordenadorias de Planejamento em Saúde, do interior e da região metropolitana de São Paulo, do Conselho de Secretários Municipais de Saúde de São Paulo e do Instituto de Saúde, culminou na criação de um aplicativo denominado SISVAN (SP). Este, consistia em um sistema destinado à coleta de informações, processamento e análise de dados nutricionais de crianças menores de 5 anos $^{6}$.

A partir de 2002 iniciou-se um processo de reestruturação e informatização da vigilância nutricional, atendendo a reivindicações dos estados e municípios que tiveram início no ano 2000 e que apontavam a necessidade urgente de uniformização do sistema em todo o território nacional $^{4}$. O processo de informatização da vigilância nutricional foi concluído em 2004 sendo implantado em diversos municípios brasileiros. O programa ficou conhecido como VAN (vigilância alimentar e nutricional) municipal.

A adoção de um critério biológico para atender os segmentos mais vulneráveis da população representou na prática, a utilização do SISVAN como um mero instrumento de triagem da clientela, além de um requisito administrativo para habilitação aos programas, o que durante muitos anos dificultou sua implantação mais efetiva no país.

Com a criação do Programa Bolsa Família em 2004, o SISVAN passou a ser o principal provedor de informações sobre a saúde das famílias beneficiadas a partir do acompanhamento do crescimento e desenvolvimento infantil ${ }^{7}$. Nesse período, em paralelo, o programa VAN municipal continuou sendo alimentado por diversos municípios. A partir de 2007, foi apresentado pelo CGPAN (Coordenação-Geral de Alimentação e Nutrição) o SISVAN Web, um novo sistema informatizado da Vigilância Alimentar e Nutricional para registro de informações do estado nutricional e do consumo alimentar da população atendida pela Atenção Básica do SUS. O SISVAN Web foi criado para substituir o programa VAN municipal, desenvolvido pelo Departamento de Informática do SUS (DATASUS) em 2003. O acesso para todos os municípios foi disponibilizado a partir de janeiro de 2008. O programa apresenta interface para captação de dados do programa bolsa família, entretanto, ambos continuam operando em paralelo.

Atualmente os sistemas informatizados de vigilância alimentar e nutricional em operação 
correspondem a um conjunto de sistemas de informações alimentados em nível local, que têm como objetivo principal produzir dados contínuos sobre as condições nutricionais e alimentares da população. Estes abrangem o SISVAN Web e SISVAN módulo de gestão (Bolsa Família), sendo este último voltado especificamente para o monitoramento das condicionalidades de saúde dos beneficiários do Programa Bolsa Família.

Decorridos quatro anos da implantação do SISVAN Web, o presente estudo teve por objetivos estimar a cobertura populacional do SISVAN nos diferentes estágios de vida e avaliar seu funcionamento no estado de São Paulo.

\section{Métodos}

O presente trabalho faz parte do projeto intitulado Rede de municípios promotores da segurança alimentar e nutricional sustentável - REDESANS realizado com recursos da Financiadora de Estudos e Projetos (FINEP). O projeto tem como uma de suas metas a avaliação das ações e os desdobramentos do monitoramento nutricional no contexto do Sistema de Vigilância Alimentar e Nutricional (SISVAN) no Estado de São Paulo.

Para a seleção da amostra do estudo consideraram-se as mesorregiões do estado de São Paulo, definidas pelo Instituto Brasileiro de Geografia e Estatística. As regiões do estado foram definidas levando-se em consideração as dimensões do processo social como determinante, do quadro natural como condicionante e da rede de comunicação e de lugares como elemento da articulação espacial.

Visto que o Estado de São Paulo possui 645 municípios, considerou-se que uma amostra de $10 \%$ dos municípios seria representativa do Estado. Sendo assim, procedeu-se um sorteio aleatório, dentro de cada região, para a seleção dos 65 municípios (Quadro 1). Para calcular o número de municípios participantes em cada uma das regiões levou-se em consideração o tamanho populacional de cada um deles.

A estimativa da cobertura populacional do SISVAN nos diferentes estágios de vida (crianças, adolescentes, adultos e idosos) foi calculada a partir de dados referentes ao total de indivíduos acompanhados, obtidos a partir de relatórios públicos disponíveis nos sítios do DATASUS e do CGPAN, e de estimativas do número de usuários que utilizam o serviço público de saúde.

Considerando-se que o setor de atenção básica não dispõe de dados relativos ao número de usuários que utilizam seus serviços, a população de referência considerada foi obtida pela diferença entre o número de habitantes do município e o total de beneficiários de planos privados de saúde, ambos por fase da vida. O grupo de gestantes não foi analisado no presente estudo pelo fato de não haver dados disponíveis sobre o número de beneficiárias de planos privados de saúde.

O número de habitantes dos 65 municípios pesquisados foi obtido a partir dos dados do censo demográfico realizado em $2010^{8}$. Informações disponibilizadas pela Agência Nacional de Saúde Suplementar (ANS) possibilitaram a identificação do total de indivíduos, por faixa etária e sexo, vinculados a planos privados de saúde referente ao ano de $2010^{9}$.

A partir dos relatórios anuais gerados pelo SISVAN Web ${ }^{10}$, consolidados semanalmente, foi possível obter informações sobre o número de indivíduos, por fase da vida, que são acompanhados pelo SISVAN em cada um dos municípios da pesquisa. Foram utilizados dados originados dos relatórios SISVAN Web, SISVAN módulo de gestão (Bolsa Família), doravante denominado SISVAN Bolsa Família (SISVAN BF), e a soma de ambos (SISVAN Web + SISVAN Bolsa Família), doravante denominado TODOS. A fragmentação das informações foi importante para identificar os municípios que preenchem somente as informações referentes ao SISVAN Bolsa Família, criado para acompanhar o registro das condi-cionalidades da saúde do Programa Bolsa Família, avaliando o acesso desse público às ações básicas de saúde.

Foram gerados relatórios anuais e mensais (janeiro a dezembro), ambos referentes ao ano de 2010. Os relatórios mensais foram gerados tanto para estimar a cobertura populacional quanto para se obter o número médio de consultas anual, já que a soma mês a mês dos usuários acompanhados pelo sistema contempla aqueles indivíduos que tiveram mais de um registro anual. Isso ocorre porque se o indivíduo tiver sido avaliado no mês de fevereiro, por exemplo, e for submetido à nova avaliação no mês de outubro, ele será computado duas vezes na soma mensal. Já o relatório anual considera sempre o resultado da última avaliação, ou seja, o indivíduo é computado apenas uma vez, independentemente do número de avaliações realizadas ao longo do ano. Sendo assim, o valor da soma mês a mês dos usuários acompanhados poderá ser maior que o valor anual caso ele tenha realizado mais de uma consulta. Para o cálculo do número médio de avaliações anuais por aqueles que tenham sido avalia- 
Quadro 1. Relação dos municípios integrantes da amostra e suas respectivas regiões.

\begin{tabular}{|c|c|c|}
\hline $\begin{array}{l}\text { No da } \\
\text { região }\end{array}$ & $\begin{array}{c}\text { Região } \\
\text { administrativa }\end{array}$ & Municípios \\
\hline 1 & Presidente Prudente & $\begin{array}{l}\text { Alfredo Marcondes, Martinópolis, Panorama, Presidente Prudente, } \\
\text { Presidente Venceslau }\end{array}$ \\
\hline 2 & Araçatuba & Araçatuba, Barbosa, Buritama, Penápolis \\
\hline 3 & São José do Rio Preto & $\begin{array}{l}\text { Álvares Florence, Auriflama, Catanduva, Cedral, Guaraci, Guarani D’Oeste, } \\
\text { José Bonifácio, Mirassol, São José do Rio Preto, Tanabi, Votuporanga }\end{array}$ \\
\hline 4 & Marília/Assis & Fernão, Garça, Marilia, Pompéia, Tupã \\
\hline 5 & Bauru & Balbinos, Barra Bonita, Bauru, Borebi, Duartina \\
\hline 6 & Araraquara & Matão, São Carlos \\
\hline 7 & Ribeirão Preto & $\begin{array}{l}\text { Altinópolis, Batatais, Cássia dos Coqueiros, Pitangueiras, Ribeirão Preto, } \\
\text { Sales de Oliveira, Sertãozinho }\end{array}$ \\
\hline 8 & Itapetininga & Buri, Itaóca, Itararé, Nova Campina \\
\hline 9 & Piracicaba & Iracemápolis, Leme, Rio Claro \\
\hline 10 & Campinas & Campinas, Hortolândia, Itobi, Jaguariúna, Pedra Bela \\
\hline 11 & Registro & Iguape, Registro \\
\hline 12 & Sorocaba/Jundiaí & Itupeva, Itu, Sorocaba, Tuiuti \\
\hline 13 & São Paulo & Guarulhos, Juquitiba, São Lourenço da Serra, Suzano \\
\hline 14 & São José dos Campos & Canas, Caraguatatuba, Roseira, Taubaté \\
\hline
\end{tabular}

dos, dividiu-se o valor obtido a partir da soma mês a mês pelo valor anual (total de usuários avaliados naquele ano).

Nos relatórios relativos ao SISVAN Bolsa Família estão registrados somente os indivíduos beneficiários deste programa, enquanto que no relatório TODOS também estão incluídos os usuários registrados no SISVAN Web. O número real de registros do SISVAN Web foi obtido a partir da diferença entre os registros de TODOS e SISVAN Bolsa Família. Isto porque em alguns municípios havia duplicidade na informação, com o mesmo indivíduo registrado no SISVAN Web e SISVAN Bolsa Família.

A consulta aos relatórios públicos do SISVAN foi realizada somente no segundo semestre de 2011, nos meses de agosto e setembro, na tentativa de se obter dados mais atualizados, já que não há uma periodicidade regular de envio das informa- ções coletadas pelos municípios para o DATASUS.

A estimativa de cobertura do SISVAN no estado de São Paulo foi calculada para as três fontes de informação (SISVAN Web, SISVAN Bolsa Família e TODOS). Foram utilizadas estatísticas descritivas como proporções, médias e desviospadrão na apresentação dos resultados. A diferença da cobertura do sistema nas 14 regiões do estado foi avaliada a partir da análise de variância com um fator (ANOVA). As análises estatísticas foram realizadas utilizando-se o SPSS 17.0. Para a elaboração dos mapas utilizou-se o software ArcGIS 10.1 (2012) ${ }^{11}$.

\section{Resultados}

A Figura 1 apresenta a cobertura populacional do SISVAN de acordo com a origem do registro: SIS- 
Tabela 1. Cobertura populacional (\%) do SISVAN nas diferentes regiões do estado de São Paulo segundo fases da vida e origem dos registros, 2010.

\begin{tabular}{|c|c|c|c|c|c|c|c|c|c|c|c|c|}
\hline \multirow{3}{*}{ Região } & \multicolumn{12}{|c|}{ Fases da vida/ Origem do registro } \\
\hline & \multicolumn{3}{|c|}{ Crianças } & \multicolumn{3}{|c|}{ Adolescentes } & \multicolumn{3}{|c|}{ Adultos } & \multicolumn{3}{|c|}{ Idosos } \\
\hline & Todos & $\mathrm{BF}^{*}$ & Web $^{* *}$ & Todos & $\mathrm{BF}$ & Web & Todos & $\mathrm{BF}$ & Web & Todos & $\mathrm{BF}$ & Web \\
\hline 1 & 42,6 & 21,4 & 20,4 & 10,4 & 7,2 & 3,0 & 13,2 & 6,8 & 5,8 & 10,0 & 0,0 & 10,0 \\
\hline 2 & 27,0 & 20,5 & 6,3 & 6,8 & 4,5 & 4,0 & 10,0 & 5,3 & 4,5 & 11,0 & 0,0 & 11,0 \\
\hline 3 & 26,3 & 15,5 & 10,4 & 5,9 & 3,4 & 1,7 & 4,3 & 3,2 & 0,7 & 0,6 & 0,0 & 0,6 \\
\hline 4 & 17,0 & 16,4 & 0,6 & 5,6 & 5,6 & 0,0 & 5,4 & 5,2 & 0,0 & 0,0 & 0,0 & 0,0 \\
\hline 5 & 30,0 & 21,6 & 8,2 & 4,8 & 4,2 & 0,8 & 4,0 & 3,6 & 0,6 & 1,2 & 0,0 & 1,2 \\
\hline 6 & 19,0 & 12,0 & 7,0 & 2,0 & 0,5 & 1,0 & 5,5 & 0,5 & 4,5 & 0,3 & 0,0 & 0,3 \\
\hline 7 & 12,8 & 12,7 & 0,1 & 4,4 & 4,4 & 1,3 & 3,8 & 3,6 & 0,1 & 0,7 & 0,0 & 0,7 \\
\hline 8 & 24,0 & 23,2 & 0,5 & 3,7 & 3,7 & 0,0 & 5,5 & 5,3 & 0,0 & 0,1 & 0,0 & 0,1 \\
\hline 9 & 16,0 & 11,0 & 4,3 & 3,0 & 3,0 & 0,0 & 4,0 & 3,7 & 0,0 & 0,0 & 0,0 & 0,0 \\
\hline 10 & 16,6 & 13,4 & 3,4 & 4,4 & 2,8 & 2,2 & 5,6 & 3,2 & 2,2 & 5,4 & 0,0 & 5,4 \\
\hline 11 & 17,0 & 16,0 & 1,0 & 5,5 & 5,5 & 0,0 & 5,5 & 5,5 & 0,0 & 0,1 & 0,0 & 0,1 \\
\hline 12 & 18,3 & 15,5 & 2,5 & 3,3 & 3,3 & 0,5 & 4,5 & 4,3 & 2,0 & 0,0 & 0,0 & 0,0 \\
\hline 13 & 19,3 & 14,5 & 4,8 & 8,3 & 7,0 & 1,3 & 7,5 & 7,3 & 0,3 & 0,1 & 0,0 & 0,1 \\
\hline 14 & 21,3 & 17,0 & 4,0 & 5,8 & 5,8 & 1,8 & 5,0 & 5,0 & 0,5 & 0,0 & 0,0 & 0,0 \\
\hline
\end{tabular}

"SISVAN Bolsa Família * SISVAN Web.

Tabela 2. Cobertura populacional (\%) do SISVAN de acordo com o porte do município e origem do registro, Estado de São Paulo, 2010.

\begin{tabular}{lccc}
\hline \multirow{2}{*}{$\begin{array}{c}\text { Porte do } \\
\text { município }\end{array}$} & \multicolumn{3}{c}{ Cobertura (\%) } \\
\cline { 2 - 4 } & Todos & SISVAN BF & SISVAN Web \\
\hline Pequeno $^{\mathrm{a}}$ & 8,1 & 5,7 & 2,4 \\
Médio $^{\mathrm{b}}$ & 5,5 & 4,4 & 1,4 \\
Grande $^{\mathrm{c}}$ & 3,5 & 2,8 & 3,8 \\
p valor $^{\#}$ & 0,11 & 0,08 & 0,54 \\
\hline
\end{tabular}

${ }^{a}$ até 100 mil habitantes; ${ }^{\text {b }}$ de 100 a 500 mil habitantes; ${ }^{\mathrm{c}}$ mais de 500 mil habitantes. ${ }^{*}$ ANOVA.

municípios de pequeno porte a cobertura populacional para o registro TODOS e SISVAN BF é duas vezes maior quando comparados aos municípios de grande porte.

O número médio de consultas anuais, entre os avaliados, revelou-se semelhante nos diferentes estágios de vida para todas as regiões do Estado. Somente na região de Araraquara (Região 6), foram realizadas em média mais de duas consultas anuais entre as crianças, e na região de São Paulo (Região 13) mais de 4 consultas anuais entre os idosos (Tabela 3).

\section{Discussão}

As informações obtidas a partir do monitoramento nutricional realizado pelo SISVAN servem de subsídio para a tomada de decisões políticas, auxiliando no planeja-mento, monitoramento e gerenciamento de programas relacionados com a melhoria dos padrões de consumo alimentar e nutricio-nal da população. Os sistemas informatizados de vigilância alimentar e nutricional vigentes apresentam inúmeras vantagens quando comparados ao sistema anterior, destacando-se a possibilidade de desagregação da informação no nível da unidade de saúde e a rapidez com que a informação é coletada, consolidada, analisada e interpretada ${ }^{7}$.

A partir da implantação do sistema informatizado em 2007, houve um aumento importante do número de acompanhamentos em todo o país, alcançando a marca de 9 milhões em aproximadamente um ano e meio de utilização ${ }^{7}$. No entanto, mesmo com todos os esforços voltados para a ampliação e, sobretudo, qualificação da vigilância alimentar e nutricional, os resultados do presente estudo revelam que a cobertura populacional tanto do SISVAN Web quanto do SISVAN Bolsa Família no estado de São Paulo permanece muito baixa. Esta baixa cobertura pode ser atribuída tanto à ausência do registro de informações quanto à falta de regularidade no en- 
Tabela 3. Número médio de avaliações entre os indivíduos monitorados, segundo região e fases da vida, Estado de São Paulo, 2010.

\begin{tabular}{|c|c|c|c|c|}
\hline \multirow[t]{2}{*}{ Região } & Crianças & Adolescentes & Adultos & Idosos \\
\hline & \multicolumn{4}{|c|}{ Média (desvio-padrão) } \\
\hline 1 & $1,6(0,55)$ & $1,2(0,5)$ & $1,0(0,0)$ & $1,2(0,4)$ \\
\hline 2 & $1,8(0,96)$ & $1,3(0,5)$ & $1,3(0,5)$ & $1,9(1,3)$ \\
\hline 3 & $1,3(0,67)$ & $1,3(0,5)$ & $1,4(0,5)$ & $1,1(0,1)$ \\
\hline 4 & $1,0(0,0)$ & $1,0(0,0)$ & $1,0(0,0)$ & $1,0(0,0)$ \\
\hline 5 & $1,0(0,0)$ & $1,0(0,0)$ & $1,0(0,0)$ & $1,0(0,0)$ \\
\hline 6 & $2,5(2,12)$ & $1,0(0,0)$ & $1,5(0,7)$ & $1,0(0,0)$ \\
\hline 7 & $1,0(0,0)$ & $1,0(0,0)$ & $1,0(0,0)$ & $1,1(0,1)$ \\
\hline 8 & $1,0(0,0)$ & $1,0(0,0)$ & $1,0(0,0)$ & $1,0(0,0)$ \\
\hline 9 & $1,0(0,0)$ & $1,0(0,0)$ & $1,0(0,0)$ & - \\
\hline 10 & $1,0(0,0)$ & $1,0(0,0)$ & $1,0(0,0)$ & $1,5(0,2)$ \\
\hline 11 & $1,0(0,0)$ & $1,0(0,0)$ & $1,0(0,0)$ & $1,0(0,0)$ \\
\hline 12 & $1,0(0,0)$ & $1,0(0,0)$ & $1,0(0,0)$ & - \\
\hline 13 & $1,3(0,5)$ & $1,3(0,5)$ & $1,3(0,5)$ & $4,4(4,6)$ \\
\hline 14 & $1,0(0,0)$ & $1,0(0,0)$ & $1,0(0,0)$ & - \\
\hline
\end{tabular}

(-) sem registro para esta faixa etária.

vio das informações coletadas pelo município para o DATASUS, sendo que esta também não é exigida ou definida em relação à população atendida por demanda espontânea. Tais resultados também podem estar relacionados à pequena apropriação por parte dos profissionais de saúde, de metodologias de planejamento de base lo$\mathrm{cal}^{12}$, uma vez que o modelo assistencial de saúde predominante, frequentemente não valoriza linhas de atuação que utilizam a informação como suporte gerencial do nível local. Nesse contexto, o monitoramento alimentar e nutricional da população tem ficado em segundo plano frente aos demais compromissos do município.

Santana e Santos ${ }^{13}$ destacam também como possível componente causal do incipiente uso da informação como ferramenta para formulação de políticas públicas locais, a tradicional centralização dos processos de formulação e gerenciamento das políticas sociais no país, que deixa para o município apenas a função de executar algo pronto, concebido fora da sua realidade e imposto de cima para baixo.

Deve-se considerar ainda que em razão das dificuldades para a implantação do sistema em caráter universal, os municípios podem priorizar, inclusive para atender as demandas do próprio Ministério, o acompanhamento da população beneficiária de programas de suplementação alimentar, transferência de renda ou grupos populacionais residentes em áreas de grande vulnerabilidade social. Em 2008, apenas metade dos municípios brasileiros registrava informações no SISVAN Web, enquanto 95\% enviaram dados antropométricos pelo sistema informatizado do Bolsa Família ${ }^{7}$. Essa tendência foi constatada neste estudo, já que o SISVAN Bolsa Família, responsável pelo registro das condicionalidades da saúde dos beneficiários do Programa, apresentou maior cobertura populacional comparado ao SISVAN Web. Também em relação ao estágio de vida, verificou-se uma preponderância de registros do estado nutricional entre crianças, fase considerada prioritária por sua maior vulnerabilidade social. A partir de um levantamento nacional utilizando dados do SISVAN Web em 2008, Coutinho et al. ${ }^{7}$ também verificaram a predominância de registros para crianças e adultos.

Merece destaque o registro inexpressivo de dados relativos à população idosa pelo SISVAN Web, sendo que mais da metade das regiões estudadas não apresentaram dados de monitoramento nutricional para esse grupo populacional. Esse resultado corrobora os achados de Coutinho et al. ${ }^{7}$, que também identificaram um número de registros bastante inferior para a população idosa, quando comparado a crianças, adolescentes, adultos e gestantes. Tal constatação gera preocupação, sobretudo por se tratar do segmento da população que mais cresce no país. Além disso, é comum nessa fase da vida haver o retorno de alguns problemas da infância (desnutrição, diarreias e infecções), além das doenças acumuladas no percurso da vida adulta. 
A análise da cobertura populacional do SISVAN Todos (SISVAN Web + SISVAN Bolsa Família), considerando o porte do município, revelou que os municípios de pequeno porte registram com maior frequência as informações nutricionais da população atendida pela atenção básica. Esse resultado pode ser atribuído ao menor número de unidades de saúde existentes nos municípios menores, o que facilitaria o treinamento de funcionários para coleta, digitação e envio das informações, facilitando consequentemente a própria gestão do sistema.

Embora as diretrizes do sistema recomendem que a periodicidade de registro dos dados de crianças siga o calendário do acompanhamento do crescimento e desenvolvimento infantil, totalizando 7 avaliações no primeiro ano de vida ${ }^{14}$, observou-se que em 9 das 14 regiões analisadas, a média foi de apenas um registro anual. Evidentemente deve-se considerar que neste estudo estão incluídas no grupo de crianças aquelas com idade entre zero e 10 anos e não somente os menores de um ano. Em relação as demais fases do curso da vida, para as quais sugere-se, no mínimo, um registro anual ${ }^{12}$, o número médio de avaliações observado no estado de São Paulo coincide com as recomendações do Ministério da Saúde, com exceção das regiões de Piracicaba (região 9), Sorocaba/Jundiaí (região 12) e São José dos Campos (região 14), que não apresentaram registros para a população de idosos.

\section{Conclusão}

Apesar dos esforços empreendidos e das estratégias adotadas pelo governo visando à ampliação e a qualificação da vigilância alimentar e nutricional, os resultados do presente estudo revelam que o monitoramento nutricional no estado de São Paulo ainda deixa muito a desejar. Percebese que a exigência formal de implantação do SISVAN não foi suficiente para garantir a geração e a utilização local de informações nos municípios, de forma que a incorporação desta prática nos serviços de saúde demanda mudanças que não dependem somente de normas. Problemas operacionais somados à falta de compromisso político de alguns gestores poderiam explicar a baixa cobertura populacional do sistema.

Este cenário compromete a utilização do SISVAN na produção de mudanças efetivas nas políticas de alimentação e nutrição. Além disso, a subutilização do sistema não condiz com o estágio de desenvolvimento econômico alcançado pelo estado, que possui atualmente o maior PIB do País.

Considerando que já estão disponíveis desde 2007 sistemas informatizados capazes de garantir agilidade na coleta, processamento, análise e interpretação das informações nutricionais da população, os esforços do governo deveriam ser direcionados para a conscientização dos gestores e profissionais de saúde sobre a importância dos dados de monitoramento nutricional para subsidiar decisões para o planejamento de políticas, gerenciamento e avaliação de programas de saúde e nutrição.

A capacitação de recursos humanos para a coleta de dados e registro das informações com maior qualidade, bem como o envio dessas informações com periodicidade regular para o DATASUS também podem contribuir para aumentar a confiabilidade da base de dados gerada pelo SISVAN.

\section{Colaboradores}

CC Enes trabalhou na análise estatística, na interpretação dos dados e redação do artigo, $\mathrm{H}$ Loiola participou da coleta dos dados nas bases oficiais e redação do artigo, MRM de Oliveira participou da concepção e delineamento do trabalho e revisão crítica. 


\section{Referências}

1. Oliveira CM, Casanova AO. Vigilância da saúde no espaço de práticas da atenção básica. Cien Saude Colet 2009; 14(3):929-936.

2. Manson JB, Habitch JP, Tabataba H, Valverde V. Vigilância nutricional. Genebra: WHO; 1984.

3. Brasil. Ministério da Saúde (MS). Secretaria de Atenção à Saúde. Departamento de Atenção Básica. Política Nacional de Alimentação e Nutrição. 2a ed. rev. Brasília: MS; 2008.

4. Brasil. Ministério da Saúde (MS). Secretaria de Atenção Básica. Departamento de Atenção Básica. Indicadores de vigilância alimentar e nutricional. Brasília: MS; 2009.

5. Engstrom EM, organizador. SISVAN: instrumento para o combate aos distúrbios nutricionais em serviços de saúde: diagnóstico nutricional. $2^{\mathrm{a}}$ ed. Rio de Janeiro: Fiocruz; 2002.

6. Venâncio SI, Levy RB, Saldiva RSDM, Mondini L, Stefanini MLR. Sistema de vigilância alimentar e nutricional no Estado de São Paulo, Brasil: experiência da implantação e avaliação do estado nutricional de crianças. Rev Bras Saúde Mater Infant 2007; $7(2): 213-220$.

7. Coutinho JG, Cardoso AJC, Toral N, Silva ACF, Ubarana JA, Aquino KKNC, Nilson EAF, Fagundes A, Vasconcellos AB. A organização da Vigilância Alimentar e Nutricional no Sistema Único de Saúde: histórico e desafios atuais. Rev Bras Epidemiol 2009; 12(4):688-699.

8. Instituto Brasileiro de Geografia e Estatística (IBGE). Censo demográfico 2010. Dados populacionais dos municípios. [Internet]. [acessado 2011 ago 3]. Disponível em: http://www.ibge.gov.br/cidadesat/

9. Departamento de Informática do Serviço Único de Saúde (DATASUS). Informações de Saúde SuplementarBeneficiários. [Internet]. [acessado 2011 ago 9]. Disponível em: < http://www2.datasus.gov.br/DATASUS
10. Sistema de Vigilância Alimentar e Nutricional (SISVAN). Dados de registros no sistema. [Internet]. [acessado 2011 ago 11]. Disponível em: http:// nutricao.saude.gov.br/SISVAN.php

11. Environmental Systems Research Institute (ESRI). Software ArcGIS 10.1. Califórnia, USA: ESRI; 2012. [Internet]. [acessado 2012 nov 6] Disponível em: http://www.esri.com/software/arcgis/index.html

12. Teixeira CF. Planejamento e programação situacional em distritos sanitários: metodologia e organização. In: Mendes EV, organizador. Distrito Sanitário: o processo social de mudança das práticas sanitárias do Sistema Único de Saúde. São Paulo: Hucitec; 1995.

13. Santana LAA, Santos SMC. Sistema de Vigilância Alimentar e Nutricional na implantação do Programa Leite é Saúde: avaliação em municípios baianos. Rev Nutr 2004; 17(3):283-290.

14. Brasil. Ministério da Saúde. Secretaria de Atenção à Saúde. Departamento de Atenção Básica. Protocolos do Sistema de Vigilância Alimentar e Nutricional - SISVAN na assistência à saúde. Brasília; 2008.

Artigo apresentado em 02/05/2013

Aprovado em 28/07/2013

Versão final apresentada em 08/08/2013 